\title{
On a combinatorial problem of Asmus Schmidt
}

\author{
W. Zudilin* \\ Department of Mechanics and Mathematics \\ Moscow Lomonosov State University \\ Vorobiovy Gory, GSP-2, Moscow 119992 RUSSIA \\ URL: http://wain.mi.ras.ru/index.html \\ E-mail address: wadim@ips.ras.ru
}

Submitted: Dec 29, 2003; Accepted: Feb 26, 2004; Published: Mar 9, 2004.

MR Subject Classifications: 11B65, 33C20.

\begin{abstract}
For any integer $r \geq 2$, define a sequence of numbers $\left\{c_{k}^{(r)}\right\}_{k=0,1, \ldots}$, independent of the parameter $n$, by

$$
\sum_{k=0}^{n}\left(\begin{array}{l}
n \\
k
\end{array}\right)^{r}\left(\begin{array}{c}
n+k \\
k
\end{array}\right)^{r}=\sum_{k=0}^{n}\left(\begin{array}{l}
n \\
k
\end{array}\right)\left(\begin{array}{c}
n+k \\
k
\end{array}\right) c_{k}^{(r)}, \quad n=0,1,2, \ldots
$$
\end{abstract}

We prove that all the numbers $c_{k}^{(r)}$ are integers.

\section{Stating the problem}

The following curious problem was stated by A. L. Schmidt in [5] in 1992.

Problem 1. For any integer $r \geq 2$, define a sequence of numbers $\left\{c_{k}^{(r)}\right\}_{k=0,1, \ldots}$, independent of the parameter $n$, by

$$
\sum_{k=0}^{n}\left(\begin{array}{l}
n \\
k
\end{array}\right)^{r}\left(\begin{array}{c}
n+k \\
k
\end{array}\right)^{r}=\sum_{k=0}^{n}\left(\begin{array}{l}
n \\
k
\end{array}\right)\left(\begin{array}{c}
n+k \\
k
\end{array}\right) c_{k}^{(r)}, \quad n=0,1,2, \ldots
$$

Is it then true that all the numbers $c_{k}^{(r)}$ are integers?

${ }^{*}$ The work is supported by an Alexander von Humboldt research fellowship and partially supported by grant no. 03-01-00359 of the Russian Foundation for Basic Research. 
An affirmative answer for $r=2$ was given in 1992 (but published a little bit later), independently, by Schmidt himself [6] and by V. Strehl [7]. They both proved the following explicit expression:

$$
c_{n}^{(2)}=\sum_{j=0}^{n}\left(\begin{array}{l}
n \\
j
\end{array}\right)^{3}=\sum_{j}\left(\begin{array}{l}
n \\
j
\end{array}\right)^{2}\left(\begin{array}{c}
2 j \\
n
\end{array}\right), \quad n=0,1,2, \ldots,
$$

which was observed experimentally by W. Deuber, W. Thumser and B. Voigt. In fact, Strehl used in [7] the corresponding identity as a model for demonstrating various proof techniques for binomial identities. He also proved an explicit expression for the sequence $c_{n}^{(3)}$, thus answering Problem 1 affirmatively in the case $r=3$. But for this case Strehl had only one proof based on Zeilberger's algorithm of creative telescoping. Problem 1 was restated in [3], Exercise (!) 114 on p. 256, with an indication (on p. 549) that H. Wilf had shown the desired integrality of $c_{n}^{(r)}$ for any $r$ but only for any $n \leq 9$.

We recall that the first non-trivial case $r=2$ is deeply related to the famous Apéry numbers $\sum_{k}\left(\begin{array}{c}n \\ k\end{array}\right)^{2}\left(\begin{array}{c}n+k \\ k\end{array}\right)^{2}$, the denominators of rational approximations to $\zeta(3)$. These numbers satisfy a 2nd-order polynomial recursion discovered by R. Apéry in 1978, while an analogous recursion (also 2nd-order and polynomial) for the numbers (2) was indicated by J. Franel already in 1894 .

The aim of this paper is to give an answer in the affirmative to Problem 1 (Theorem 1) by deriving explicit expressions for the numbers $c_{n}^{(r)}$, and also to prove a stronger result (Theorem 2) conjectured in [7], Section 4.2.

Theorem 1. The answer to Problem 1 is affirmative. In particular, we have the explicit expressions

$$
\begin{aligned}
& c_{n}^{(4)}=\sum_{j}\left(\begin{array}{c}
2 j \\
j
\end{array}\right)^{3}\left(\begin{array}{l}
n \\
j
\end{array}\right) \sum_{k}\left(\begin{array}{c}
k+j \\
k-j
\end{array}\right)\left(\begin{array}{c}
j \\
n-k
\end{array}\right)\left(\begin{array}{l}
k \\
j
\end{array}\right)\left(\begin{array}{c}
2 j \\
k-j
\end{array}\right), \\
& c_{n}^{(5)}=\sum_{j}\left(\begin{array}{c}
2 j \\
j
\end{array}\right)^{4}\left(\begin{array}{l}
n \\
j
\end{array}\right)^{2} \sum_{k}\left(\begin{array}{l}
k+j \\
k-j
\end{array}\right)^{2}\left(\begin{array}{c}
2 j \\
n-k
\end{array}\right)\left(\begin{array}{c}
2 j \\
k-j
\end{array}\right),
\end{aligned}
$$

and in general for $s=1,2, \ldots$

$$
\begin{aligned}
c_{n}^{(2 s)}= & \sum_{j}\left(\begin{array}{c}
2 j \\
j
\end{array}\right)^{2 s-1}\left(\begin{array}{c}
n \\
j
\end{array}\right) \sum_{k_{1}}\left(\begin{array}{c}
j \\
n-k_{1}
\end{array}\right)\left(\begin{array}{l}
k_{1} \\
j
\end{array}\right)\left(\begin{array}{c}
k_{1}+j \\
k_{1}-j
\end{array}\right) \sum_{k_{2}}\left(\begin{array}{c}
2 j \\
k_{1}-k_{2}
\end{array}\right)\left(\begin{array}{l}
k_{2}+j \\
k_{2}-j
\end{array}\right)^{2} \ldots \\
& \times \sum_{k_{s-1}}\left(\begin{array}{c}
2 j \\
k_{s-2}-k_{s-1}
\end{array}\right)\left(\begin{array}{c}
k_{s-1}+j \\
k_{s-1}-j
\end{array}\right)^{2}\left(\begin{array}{c}
2 j \\
k_{s-1}-j
\end{array}\right), \\
c_{n}^{(2 s+1)}= & \sum_{j}\left(\begin{array}{c}
2 j \\
j
\end{array}\right)^{2 s}\left(\begin{array}{c}
n \\
j
\end{array}\right)^{2} \sum_{k_{1}}\left(\begin{array}{c}
2 j \\
n-k_{1}
\end{array}\right)\left(\begin{array}{l}
k_{1}+j \\
k_{1}-j
\end{array}\right)^{2} \sum_{k_{2}}\left(\begin{array}{c}
2 j \\
k_{1}-k_{2}
\end{array}\right)\left(\begin{array}{c}
k_{2}+j \\
k_{2}-j
\end{array}\right)^{2} \ldots \\
& \times \sum_{k_{s-1}}\left(\begin{array}{c}
2 j \\
k_{s-2}-k_{s-1}
\end{array}\right)\left(\begin{array}{c}
k_{s-1}+j \\
k_{s-1}-j
\end{array}\right)^{2}\left(\begin{array}{c}
2 j \\
k_{s-1}-j
\end{array}\right),
\end{aligned}
$$

where $n=0,1,2, \ldots$. 


\section{$2 \quad$ Very-well-poised preliminaries}

The right-hand side of (1) defines the so-called Legendre transform of the sequence $\left\{c_{k}^{(r)}\right\}_{k=0,1, \ldots .}$. In general, if

$$
a_{n}=\sum_{k=0}^{n}\left(\begin{array}{l}
n \\
k
\end{array}\right)\left(\begin{array}{c}
n+k \\
k
\end{array}\right) c_{k}=\sum_{k=0}^{n}\left(\begin{array}{c}
2 k \\
k
\end{array}\right)\left(\begin{array}{c}
n+k \\
n-k
\end{array}\right) c_{k},
$$

then by the well-known relation for inverse Legendre pairs one has

$$
\left(\begin{array}{c}
2 n \\
n
\end{array}\right) c_{n}=\sum_{k}(-1)^{n-k} d_{n, k} a_{k}
$$

where

$$
d_{n, k}=\left(\begin{array}{c}
2 n \\
n-k
\end{array}\right)-\left(\begin{array}{c}
2 n \\
n-k-1
\end{array}\right)=\frac{2 k+1}{n+k+1}\left(\begin{array}{c}
2 n \\
n-k
\end{array}\right) .
$$

Therefore, putting

$$
t_{n, j}^{(r)}=\sum_{k=j}^{n}(-1)^{n-k} d_{n, k}\left(\begin{array}{c}
k+j \\
k-j
\end{array}\right)^{r}
$$

we obtain

$$
\left(\begin{array}{c}
2 n \\
n
\end{array}\right) c_{n}^{(r)}=\sum_{j=0}^{n}\left(\begin{array}{c}
2 j \\
j
\end{array}\right)^{r} t_{n, j}^{(r)}
$$

The case $r=1$ of Problem 1 is trivial (that is why it is not included in the statement of the problem), while the cases $r=2$ and $r=3$ are treated in [6], [7] using the fact that $t_{n, j}^{(2)}$ and $t_{n, j}^{(3)}$ have a closed form. Namely, it is easy to show by Zeilberger's algorithm of creative telescoping [4] that the latter sequences, indexed by either $n$ or $j$, satisfy simple 1st-order polynomial recursions. Unfortunately, this argument does not exist for $r \geq 4$.

V. Strehl observed in [7], Section 4.2, that the desired integrality would be a consequence of the divisibility of the product $\left(\begin{array}{c}2 j \\ j\end{array}\right)^{r} \cdot t_{n, j}^{(r)}$ by $\left(\begin{array}{c}2 n \\ n\end{array}\right)$ for all $j, 0 \leq j \leq n$. He conjectured a much stronger property, which we are now able to prove.

Theorem 2. The numbers $\left(\begin{array}{c}2 n \\ n\end{array}\right)^{-1}\left(\begin{array}{c}2 j \\ j\end{array}\right) t_{n, j}^{(r)}$ are integers.

Our general strategy for proving Theorem 2 (and hence Theorem 1) is as follows: rewrite (5) in a hypergeometric form and apply suitable summation and transformation formulae (Propositions 1 and 2 below).

Changing $l$ to $n-k$ in (5) we obtain

$$
t_{n, j}^{(r)}=\sum_{l \geq 0}(-1)^{l} \frac{2 n-2 l+1}{2 n-l+1}\left(\begin{array}{c}
2 n \\
l
\end{array}\right)\left(\begin{array}{c}
n-l+j \\
n-l-j
\end{array}\right)^{r},
$$


where the series on the right terminates. It is convenient to write all such terminating sums simply as $\sum_{l}$, which is, in fact, a standard convention (see, e.g., [4]). The ratio of two consecutive terms in the latter sum is equal to

$$
\frac{-(2 n+1)+l}{1+l} \cdot \frac{-\frac{1}{2}(2 n-1)+l}{-\frac{1}{2}(2 n+1)+l} \cdot\left(\frac{-(n-j)+l}{-(n+j)+l}\right)^{r}
$$

hence

$$
t_{n, j}^{(r)}=\left(\begin{array}{l}
n+j \\
n-j
\end{array}\right)^{r} \cdot{ }_{r+2} F_{r+1}\left(\begin{array}{r}
-(2 n+1),-\frac{1}{2}(2 n-1),-(n-j), \ldots,-(n-j) \\
-\frac{1}{2}(2 n+1),-(n+j), \ldots,-(n+j)
\end{array} \mid 1\right)
$$

is a very-well-poised hypergeometric series. (We refer the reader to the book [2] for all necessary hypergeometric definitions. We will omit the argument $z=1$ in further discussions.)

The following two classical results-Dougall's summation of a ${ }_{5} F_{4}(1)$-series (proved in 1907) and Whipple's transformation of a ${ }_{7} F_{6}(1)$-series (proved in 1926) - will be required to treat the cases $r=3,4,5$ of Theorems 1 and 2 .

Proposition 1 ([2], Section 4.3). We have

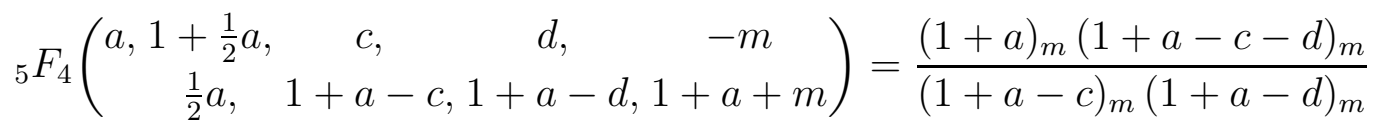

and

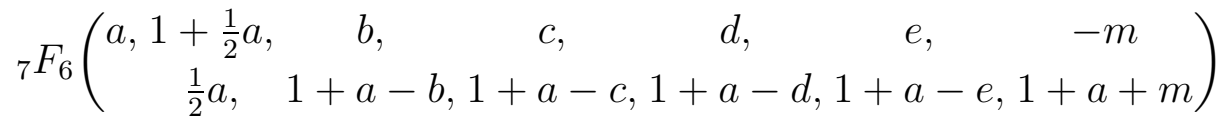

$$
\begin{aligned}
& =\frac{(1+a)_{m}(1+a-d-e)_{m}}{(1+a-d)_{m}(1+a-e)_{m}} \cdot{ }_{4} F_{3}\left(\begin{array}{c}
1+a-b-c, d, e,-m \\
1+a-b, 1+a-c, d+e-a-m
\end{array}\right),
\end{aligned}
$$

where $m$ is a non-negative integer, and $(\cdot)$ denotes Pochhammer's symbol.

An application of (7) gives (without creative telescoping)

$$
t_{n, j}^{(3)}=\left(\begin{array}{c}
n+j \\
n-j
\end{array}\right)^{3} \cdot \frac{(-2 n)_{n-j}(-2 n+2(n-j))_{n-j}}{(-2 n+(n-j))_{n-j}^{2}}=\frac{(2 n) !}{(3 j-n) !(n-j) !^{3}},
$$

which is exactly the expression obtained in [7], Section 4.2. Therefore, from (6) we have the explicit expression

$$
c_{n}^{(3)}=\left(\begin{array}{c}
2 n \\
n
\end{array}\right)^{-1} \sum_{j}\left(\begin{array}{c}
2 j \\
j
\end{array}\right)^{3} \frac{(2 n) !}{(3 j-n) !(n-j) ! 3}=\sum_{j}\left(\begin{array}{c}
2 j \\
j
\end{array}\right)^{2}\left(\begin{array}{c}
2 j \\
n-j
\end{array}\right)\left(\begin{array}{c}
n \\
j
\end{array}\right)^{2} .
$$


For the case $r=5$, we are able to apply the transformation (8):

$$
\begin{aligned}
t_{n, j}^{(5)}= & \left(\begin{array}{c}
n+j \\
n-j
\end{array}\right)^{5} \cdot \frac{(-2 n)_{n-j}(-2 n+2(n-j))_{n-j}}{(-2 n+(n-j))_{n-j}^{2}} \\
& \times{ }_{4} F_{3}\left(\begin{array}{c}
-2 j,-(n-j),-(n-j),-(n-j) \\
-(n+j),-(n+j), 3 j-n+1
\end{array}\right) \\
= & \left(\begin{array}{c}
n+j \\
n-j
\end{array}\right)^{2} \frac{(2 n) !}{(3 j-n) !(n-j) !^{3}} \sum_{l} \frac{(-2 j)_{l}(-(n-j))_{l}^{3}}{l !(-(n+j))_{l}^{2}(3 j-n+1)_{l}} \\
= & \frac{(2 n) !}{(2 j) !(n-j) !^{2}} \sum_{l}\left(\begin{array}{l}
n-l+j \\
n-l-j
\end{array}\right)^{2}\left(\begin{array}{c}
2 j \\
l
\end{array}\right)\left(\begin{array}{c}
2 j \\
n-l-j
\end{array}\right) \\
= & \frac{(2 n) !}{(2 j) !(n-j) !^{2}} \sum_{k}\left(\begin{array}{l}
k+j \\
k-j
\end{array}\right)^{2}\left(\begin{array}{c}
2 j \\
n-k
\end{array}\right)\left(\begin{array}{c}
2 j \\
k-j
\end{array}\right),
\end{aligned}
$$

hence

$$
\left(\begin{array}{c}
2 n \\
n
\end{array}\right)^{-1}\left(\begin{array}{c}
2 j \\
j
\end{array}\right) t_{n, j}^{(5)}=\left(\begin{array}{c}
n \\
j
\end{array}\right)^{2} \sum_{k}\left(\begin{array}{l}
k+j \\
k-j
\end{array}\right)^{2}\left(\begin{array}{c}
2 j \\
n-k
\end{array}\right)\left(\begin{array}{c}
2 j \\
k-j
\end{array}\right)
$$

are integers and from (6) we derive formula (4).

To proceed in the case $r=4$, we apply the version of formula (8) with $b=(1+a) / 2$ (so that the series on the left reduces to $a_{6} F_{5}(1)$-very-well-poised series):

$$
\begin{aligned}
t_{n, j}^{(4)}= & \left(\begin{array}{c}
n+j \\
n-j
\end{array}\right)^{4} \cdot \frac{(-2 n)_{n-j}(-2 n+2(n-j))_{n-j}}{(-2 n+(n-j))_{n-j}^{2}} \\
& \times{ }_{4} F_{3}\left(\begin{array}{c}
-j,-(n-j),-(n-j),-(n-j) \\
-n,-(n+j), 3 j-n+1
\end{array}\right) \\
= & \left(\begin{array}{c}
n+j \\
n-j
\end{array}\right) \frac{(2 n) !}{(3 j-n) !(n-j) ! 3} \sum_{l} \frac{(-j)_{l}(-(n-j))_{l}^{3}}{l !(-n)_{l}(-(n+j))_{l}(3 j-n+1)_{l}} \\
= & \frac{(2 n) ! j !}{n !(n-j) !(2 j) !} \sum_{l}\left(\begin{array}{c}
n-l+j \\
n-l-j
\end{array}\right)\left(\begin{array}{c}
j \\
l
\end{array}\right)\left(\begin{array}{c}
n-l \\
j
\end{array}\right)\left(\begin{array}{c}
2 j \\
n-l-j
\end{array}\right) \\
= & \frac{(2 n) ! j !}{n !(n-j) !(2 j) !} \sum_{k}\left(\begin{array}{l}
k+j \\
k-j
\end{array}\right)\left(\begin{array}{c}
j \\
n-k
\end{array}\right)\left(\begin{array}{c}
k \\
j
\end{array}\right)\left(\begin{array}{c}
2 j \\
k-j
\end{array}\right),
\end{aligned}
$$

from which, again, $\left(\begin{array}{c}2 n \\ n\end{array}\right)^{-1}\left(\begin{array}{c}2 j \\ j\end{array}\right) t_{n, j}^{(4)} \in \mathbb{Z}$ and we arrive at formula (3).

\section{Andrews's multiple transformation}

It seems that 'classical' hypergeometric identities can cover only the cases ${ }^{1} r=2,3,4,5$ of Theorems 1 and 2. In order to prove the theorems in full generality, we will require

\footnotetext{
${ }^{1}$ This is not really true since Andrews's 'non-classical' identity below is a consequence of very classical Whipple's transformation and the Pfaff-Saalschütz formula.
} 
a multiple generalization of Whipple's transformation (8). The required generalization is given by G. E. Andrews in [1], Theorem 4. After making the passage $q \rightarrow 1$ in Andrews's theorem, we arrive at the following result.

Proposition 2. For $s \geq 1$ and $m$ a non-negative integer,

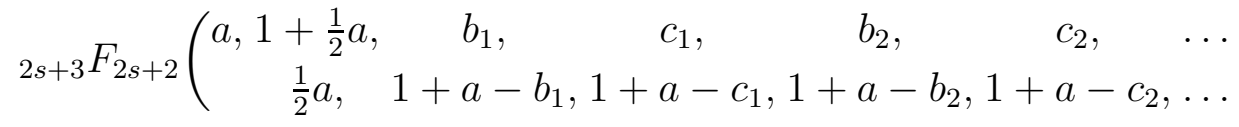

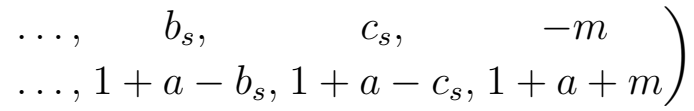

$$
\begin{aligned}
& =\frac{(1+a)_{m}\left(1+a-b_{s}-c_{s}\right)_{m}}{\left(1+a-b_{s}\right)_{m}\left(1+a-c_{s}\right)_{m}} \sum_{l_{1} \geq 0} \frac{\left(1+a-b_{1}-c_{1}\right)_{l_{1}}\left(b_{2}\right)_{l_{1}}\left(c_{2}\right)_{l_{1}}}{l_{1} !\left(1+a-b_{1}\right)_{l_{1}}\left(1+a-c_{1}\right)_{l_{1}}} \\
& \times \sum_{l_{2} \geq 0} \frac{\left(1+a-b_{2}-c_{2}\right)_{l_{2}}\left(b_{3}\right)_{l_{1}+l_{2}}\left(c_{3}\right)_{l_{1}+l_{2}}}{l_{2} !\left(1+a-b_{2}\right)_{l_{1}+l_{2}}\left(1+a-c_{2}\right)_{l_{1}+l_{2}}} \cdots \\
& \times \sum_{l_{s-1} \geq 0} \frac{\left(1+a-b_{s-1}-c_{s-1}\right){ }_{l_{s-1}}\left(b_{s}\right)_{l_{1}+\cdots+l_{s-1}}\left(c_{s}\right)_{l_{1}+\cdots+l_{s-1}}}{l_{s-1} !\left(1+a-b_{s-1}\right)_{l_{1}+\cdots+l_{s-1}}\left(1+a-c_{s-1}\right)_{l_{1}+\cdots+l_{s-1}}} \\
& \times \frac{(-m)_{l_{1}+\cdots+l_{s-1}}}{\left(b_{s}+c_{s}-a-m\right)_{l_{1}+\cdots+l_{s-1}}} .
\end{aligned}
$$

Proof of Theorem 2. As in Section 2, we will distinguish the cases corresponding to the parity of $r$.

If $r=2 s+1$, then setting $a=-(2 n+1)$ and $b_{1}=c_{1}=\cdots=b_{s}=c_{s}=-m=-(n-j)$ in Proposition 2 we obtain

$$
\begin{aligned}
& t_{n, j}^{(2 s+1)}=\left(\begin{array}{c}
n+j \\
n-j
\end{array}\right)^{2 s-2} \frac{(2 n) !}{(3 j-n) !(n-j) !} \sum_{l_{1}}\left(\begin{array}{c}
2 j \\
l_{1}
\end{array}\right)\left(\frac{(-(n-j))_{l_{1}}}{(-(n+j))_{l_{1}}}\right)^{2} \\
& \times \sum_{l_{2}}\left(\begin{array}{c}
2 j \\
l_{2}
\end{array}\right)\left(\frac{(-(n-j))_{l_{1}+l_{2}}}{(-(n+j))_{l_{1}+l_{2}}}\right)^{2} \ldots \\
& \times \sum_{l_{s-1}}\left(\begin{array}{c}
2 j \\
l_{s-1}
\end{array}\right)\left(\frac{(-(n-j))_{l_{1}+\cdots+l_{s-1}}}{(-(n+j))_{l_{1}+\cdots+l_{s-1}}}\right)^{2} \\
& \times \frac{(-1)^{l_{1}+\cdots+l_{s-1}}(-(n-j))_{l_{1}+\cdots+l_{s-1}}}{(3 j-n+1)_{l_{1}+\cdots+l_{s-1}}} \\
& =\frac{(2 n) !}{(2 j) !(n-j) !^{2}} \sum_{l_{1}}\left(\begin{array}{l}
2 j \\
l_{1}
\end{array}\right)\left(\begin{array}{l}
n-l_{1}+j \\
n-l_{1}-j
\end{array}\right)^{2} \sum_{l_{2}}\left(\begin{array}{l}
2 j \\
l_{2}
\end{array}\right)\left(\begin{array}{l}
n-l_{1}-l_{2}+j \\
n-l_{1}-l_{2}-j
\end{array}\right)^{2} \ldots \\
& \times \sum_{l_{s-1}}\left(\begin{array}{c}
2 j \\
l_{s-1}
\end{array}\right)\left(\begin{array}{l}
n-l_{1}-\cdots-l_{s-1}+j \\
n-l_{1}-\cdots-l_{s-1}-j
\end{array}\right)^{2} \cdot\left(\begin{array}{c}
2 j \\
n-l_{1}-\cdots-l_{s-1}-j
\end{array}\right) .
\end{aligned}
$$

If $r=2 s$, we apply Proposition 2 with the choice $a=-(2 n+1), b_{1}=(a+1) / 2=-n$ 
and $c_{1}=b_{2}=\cdots=b_{s}=c_{s}=-m=-(n-j)$ :

$$
\begin{aligned}
& t_{n, j}^{(2 s)}=\left(\begin{array}{c}
n+j \\
n-j
\end{array}\right)^{2 s-3} \frac{(2 n) !}{(3 j-n) !(n-j) ! 3} \sum_{l_{1}}\left(\begin{array}{c}
j \\
l_{1}
\end{array}\right) \frac{(-(n-j))_{l_{1}}}{(-n)_{l_{1}}} \frac{(-(n-j))_{l_{1}}}{(-(n+j))_{l_{1}}} \\
& \times \sum_{l_{2}}\left(\begin{array}{c}
2 j \\
l_{2}
\end{array}\right)\left(\frac{(-(n-j))_{l_{1}+l_{2}}}{(-(n+j))_{l_{1}+l_{2}}}\right)^{2} \ldots \\
& \times \sum_{l_{s-1}}\left(\begin{array}{c}
2 j \\
l_{s-1}
\end{array}\right)\left(\frac{(-(n-j))_{l_{1}+\cdots+l_{s-1}}}{(-(n+j))_{l_{1}+\cdots+l_{s-1}}}\right)^{2} \\
& \times \frac{(-1)^{l_{1}+\cdots+l_{s-1}}(-(n-j))_{l_{1}+\cdots+l_{s-1}}}{(3 j-n+1)_{l_{1}+\cdots+l_{s-1}}} \\
& =\frac{(2 n) ! j !}{n !(n-j) !(2 j) !} \sum_{l_{1}}\left(\begin{array}{c}
j \\
l_{1}
\end{array}\right)\left(\begin{array}{c}
n-l_{1} \\
j
\end{array}\right)\left(\begin{array}{c}
n-l_{1}+j \\
n-l_{1}-j
\end{array}\right) \\
& \times \sum_{l_{2}}\left(\begin{array}{l}
2 j \\
l_{2}
\end{array}\right)\left(\begin{array}{l}
n-l_{1}-l_{2}+j \\
n-l_{1}-l_{2}-j
\end{array}\right)^{2} \cdots \\
& \times \sum_{l_{s-1}}\left(\begin{array}{c}
2 j \\
l_{s-1}
\end{array}\right)\left(\begin{array}{l}
n-l_{1}-\cdots-l_{s-1}+j \\
n-l_{1}-\cdots-l_{s-1}-j
\end{array}\right)^{2} \cdot\left(\begin{array}{c}
2 j \\
n-l_{1}-\cdots-l_{s-1}-j
\end{array}\right) .
\end{aligned}
$$

In both cases, the desired integrality

$$
\left(\begin{array}{c}
2 n \\
n
\end{array}\right)^{-1}\left(\begin{array}{c}
2 j \\
j
\end{array}\right) t_{n, j}^{(r)} \in \mathbb{Z}, \quad j=0,1, \ldots, n,
$$

clearly holds, and Theorem 2 follows.

Theorem 1 was actually proved during the proof of Theorem 2 with explicit expressions being obtained for $c_{n}^{(4)}, c_{n}^{(5)}$ and general $c_{n}^{(r)}, r \geq 2$.

We would like to conclude the paper by the following $q$-question.

Problem 2. Find and solve an appropriate q-analogue of Problem 1.

Acknowledgements. I was greatly encouraged by C. Krattenthaler to prove binomial identities by myself. I thank him for our fruitful discussions and for pointing out to me Andrews's formula. I thank J. Sondow for several suggestions that allowed me to improve the text of the paper. This work was done during a long-term visit at the Mathematical Institute of Cologne University. I thank the staff of the institute and personally P. Bundschuh for the brilliant working atmosphere I had there.

\section{References}

[1] G. E. Andrews, "Problems and prospects for basic hypergeometric functions", Theory and application of special functions (Proc. Advanced Sem., Math. Res. Center, Univ. Wisconsin, 
Madison, Wis., 1975), ed. R. A. Askey, Math. Res. Center, Univ. Wisconsin, Publ. No. 35, Academic Press, New York, 1975, pp. 191-224.

[2] W. N. Bailey, Generalized hypergeometric series, Cambridge Math. Tracts 32, Cambridge Univ. Press, Cambridge, 1935; 2nd reprinted edition, Stechert-Hafner, New York-London, 1964.

[3] R. L. Graham, D.E. Knuth, and O. Patashnik, Concrete mathematics. A foundation for computer science, 2nd edition, Addison-Wesley Publishing Company, Reading, MA, 1994.

[4] M. Petkovšek, H. S. Wilf, and D. Zeilberger, $A=B$, A. K. Peters, Ltd., Wellesley, MA, 1996.

[5] A. L. Schmidt, "Generalized $q$-Legendre polynomials", Proc. of the 7th Spanish Symposium on Orthogonal Polynomials and Applications (VII SPOA) (Granada, 1991), J. Comput. Appl. Math. 49:1-3 (1993), 243-249.

[6] A. L. Schmidt, "Legendre transforms and Apéry's sequences", J. Austral. Math. Soc. Ser. A 58:3 (1995), 358-375.

[7] V. Strehl, "Binomial identities - combinatorial and algorithmic aspects", Discrete Math. 136:1-3 (1994), 309-346. 